\title{
Features of the Behavior of a Polymer Solution Jet in Electrospinning
}

\author{
A. V. Subbotin ${ }^{a, b, *}$ \\ ${ }^{a}$ Topchiev Institute of Petrochemical Synthesis, Russian Academy of Sciences, Moscow, 119991 Russia \\ ${ }^{b}$ Frumkin Institute of Physical Chemistry and Electrochemistry, Russian Academy of Sciences, Moscow, 119071 Russia \\ *e-mail:subbotin@ips.ac.ru
}

Received September 24, 2020; revised October 26, 2020; accepted November 2, 2020

\begin{abstract}
Using the numerical analysis of the force balance equation and the rheological equation of the model of finitely extensible chains, the dynamics of a charged jet during the electrospinning of a polymer solution and the orientation of macromolecules in the jet are studied. In fairly weak electric fields, the jet always remains rectilinear, while in strong fields the straight section of the jet has a finite length, after which the motion of the jet becomes unstable. This behavior is due to the competition between inertial and viscoelastic forces, with viscoelasticity dominating in strong fields. It is found that polymer chains in the jet are strongly stretched along the flow direction.
\end{abstract}

DOI: $10.1134 / \mathrm{S} 0965545 \mathrm{X} 21020127$

\section{INTRODUCTION}

It is well known that, in strong electric fields, the menisci of polymer solutions emit thin streams, from which a fiber is formed after evaporation of the solvent [1-3]. This phenomenon is widely used to obtain a variety of fibrous materials that have found application in medicine, catalysis, creation of filters, and other fields $[3,4]$. Experiments show that the flow of a jet under the action of an electric field is characterized by a high rate of extension, which exceeds the inverse relaxation time of macromolecules, and can be accompanied by the phase transition and formation of filamentary structures in it [5-7]. In addition, the orientation of macromolecules along the jet axis occurs. Orientation is also observed in the nanofibers formed on the collector [8-10]. This is consistent with the general picture of the behavior of polymer solutions under mechanical extension, including phase separation and fiber formation [11-15]. One of the mechanisms controlling the separation of solutions under extension is a change in the balance between the attraction and repulsion of macromolecules due to their flow-induced orientation [16-18]. The same mechanism should operate during electrospinning.

Under typical conditions of the electrospinning process, the solution slowly flows out of the electrode nozzle and takes the form of a cone-shaped meniscus, the top of which emits a thin jet (Fig. 1). According to the Taylor theory, the stabilization of the cone-shaped meniscus is ensured by the action of the normal electrostatic force and capillary force [19]. However, a static cone with an apex angle of $98.6^{\circ}$, which was found by Taylor, cannot emit a jet. Experiments show that there is a fairly wide variation in the angle values [20], and the question of predicting the shape of the meniscus is still open.

The transition region between the cone and the jet plays an important role in the behavior of the jet. In this region, the mechanism of charge flow along the

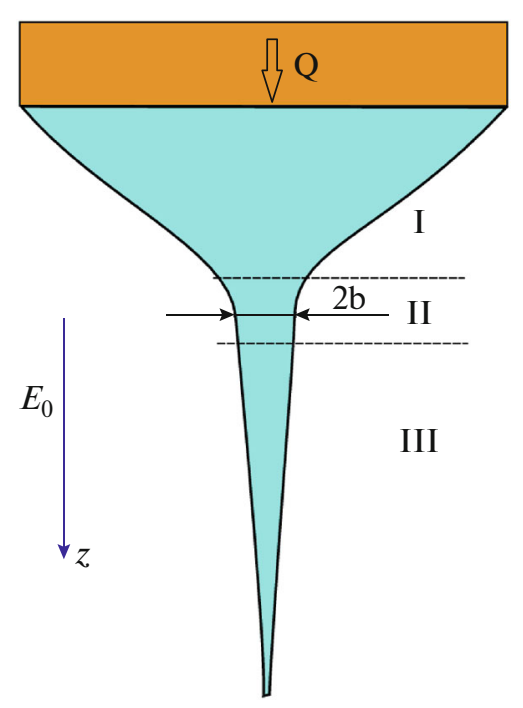

Fig. 1. Diagram illustrating the jet flow from the coneshaped meniscus (region I) during electrospinning. The radius of transition region II is $b$. The jet flow occurs in region III. Color drawings can be viewed in the electronic version. 
jet, as well as the balance of the acting forces, changes $[1,21-23]$. In addition, this region is characterized by the maximum rate of solution deformation [24-26].

The total electric current in the cone-jet system includes the ion current in the bulk $I_{\mathrm{b}}$, which leads to charging of the free surface of the liquid; the surface ion current $I_{\mathrm{s}}$ induced by the field; and the surface convective current $I_{\mathrm{c}}$ due to liquid flow:

$$
I=I_{\mathrm{b}}+I_{\mathrm{s}}+I_{\mathrm{c}} .
$$

The bulk current predominates inside the meniscus, while in the jet the total electric current is primarily determined by the surface conduction current or the convective current. In the transition region, $I_{\mathrm{b}} \simeq I_{\mathrm{c}}$ or $I_{\mathrm{b}} \simeq I_{\mathrm{s}}$.

Following Taylor's arguments, the free electric charge density $\sigma_{\mathrm{f}}$ on the jet surface in the transition region is found from the balance of capillary and normal electrostatic forces: $\sigma_{\mathrm{f}} \sim\left(\gamma \varepsilon_{0} / b\right)^{1 / 2}$, where $\gamma$ is the surface tension and $b$ is the radius of the transition region $\left(\varepsilon_{0}\right.$ is the vacuum permittivity) [1, 2]. This charge creates a local stress field $E \sim \sigma_{\mathrm{f}} / \varepsilon_{0}$. Along with this field, the field $E_{0}$ created by the electrodes acts on the liquid. If the electric field inside the transition region is mainly defined by surface charges and the current in the jet is associated with convection, then the radius of the transition region is $b=b_{1} \sim\left(Q \varepsilon_{0} / K\right)^{1 / 3}$. In this case, the electric current changes as $I \simeq 2 I_{\mathrm{b}} \sim(\gamma K Q)^{1 / 2}$ [21]. Here $Q$ is the volumetric flow rate and $K$ is the electrical conductivity coefficient. When the electric field in the transition region becomes on the order of the external field, $E \simeq E_{0}$, another mode arises, in which the radius of the transition region and the electric current are given by expressions $b=b_{2} \sim\left(\frac{Q}{K E_{0}}\right)^{2 / 7}\left(\gamma \varepsilon_{0}\right)^{1 / 7}$ and $I \sim$ $\left(\gamma \varepsilon_{0}\right)^{2 / 7}\left(K E_{0}\right)^{3 / 7} Q^{4 / 7}$, respectively [27]. For a given field $E_{0}$ at a high flow rate, the radius of the transition region is $b_{2}$, while at low flow rates the radius of the transition region is $b_{1}$. A further decrease in the liquid flow rate leads to a decrease in the convective current, so that at a very small liquid flow rate the radius of the transition region is determined by the condition $I_{\mathrm{b}} \simeq I_{\mathrm{s}}$ and is equal to $b=b_{3} \sim(\mu / K)^{2 / 3}\left(\gamma \varepsilon_{0}\right)^{1 / 3}[28$, 29 ]. Here $\mu$ is the mobility of surface charges. The current changes as $I \sim e \gamma \mu$, where $e$ is the unit charge [28].

In most electrospinning modes, the electric current mainly consists of bulk current $I_{\mathrm{b}}$ and convective current $I_{\mathrm{c}}$, while the surface conduction current $I_{\mathrm{s}}$ is fairly small. The jet dynamics in this case is controlled by the balance of the tangential electric force, on one hand, and the capillary, viscoelastic, and inertial forces, on the other hand. The contribution of the normal electric force becomes insignificant. This follows from the fact that the surface charge density of the jet decreases with decreasing radius of the jet $a$ as $\sigma_{\mathrm{f}} \sim a I / Q$ [19]. Thus, the normal electric force acting per unit area of the free surface $F_{\mathrm{n}} \sim \sigma_{\mathrm{f}}^{2} / \varepsilon_{0} \propto a^{2}$ also decreases. At the same time, the Laplace pressure $\gamma / a$ inside the jet increases and can no longer be compensated by the normal electric pressure: it is compensated by the action of the tangential electric force. To ensure a smooth change in the balance of forces in the transition region, the normal and tangential components of the electric force per unit surface area and the Laplace pressure must be of the same order of magnitude. For the steady outflow of the jet, the viscoelastic and inertial forces should not exceed the capillary forces in the transition region.

The jet shape was investigated both theoretically [27, 29-34] and using various experimental methods $[26,35,36]$. Analysis of the force balance equation showed that, when the tangential electric force and capillary force dominate, the radius of the jet decreases with distance $z$ as $a(z) \propto z^{-1}$. A similar behavior is obtained from the balance of tangential electric and viscous forces [27]. When passing from viscous to viscoelastic forces, a different behavior is predicted $a(z) \propto z^{-1 / 2}$ [35]. Finally, the balance of electric and inertial forces gives $a(z) \propto z^{-1 / 4}$ [30].

An important problem concerns determining the conditions for the stability of the jet during electrospinning. A large number of works [37-44] are devoted to this issue. The main theoretical results were obtained using linear analysis of stability. In this case, the jet was modeled by a long charged cylinder. The cases of both axially symmetric $[41,42]$ and bending perturbations of a cylindrical jet $[43,44]$ were considered.

In this work, the dynamics of a charged jet and the orientation of macromolecules in the jet are studied by the numerical analysis of the balance equation for electric, capillary, viscoelastic, and inertial forces and using the governing equation of the FENE-P model (finitely extensible nonlinear elasticity) [45], which describes the rheological behavior of a solution of polymer chains. The approach used makes it possible to reveal the role of finite extensibility in the jet dynamics under strong chain extension.

\section{JET DYNAMICS EQUATIONS}

Let us consider an axially symmetric jet of a polymer solution emitted by a conical meniscus in the direction of axis $z$ (Fig. 1). It is assumed that electrodes create an electric field of strength $E_{0}$ in direction $z$. Since the radius of the jet $a(z)$ changes slowly 
with distance $\left|\frac{d a}{d z}\right| \ll 1$, its behavior can be described using the slender body approximation [31-34]. In the stationary mode, the flow velocity in the jet and the jet stretching rate are represented as follows:

$$
v_{z}=\frac{Q}{\pi a^{2}(z)} \quad \text { and } \quad \dot{\varepsilon}=\frac{d v_{z}}{d z} \simeq-\frac{2 Q}{\pi a^{3}} \frac{d a}{d z},
$$

where $Q$ is the volume flow rate of the liquid. Further, we will use the following physical parameters: the polymer solution is characterized by density $\rho$ and surface tension $\gamma$. The electrical conductivity of ions in solution is $K$.

The FENE-P model is widely used to study the dynamics of stretching of semidilute polymer solutions $[46,47]$. It also allows one to take into account the finite extensibility of polymer coils. We will assume that the polymer chains have the full length $L$ and segment length $a_{\mathrm{s}}$, so that the total number of segments in the chain is $N=L / a_{s} \gg 1$. The dependence of chain tensile force $\mathbf{f}$ on the distance between its ends $\mathbf{R}$ is described by a nonlinear law $\mathbf{f}(\mathbf{R})=\frac{3 k_{\mathrm{B}} T}{\left\langle\mathbf{R}^{2}\right\rangle_{0}} \frac{\mathbf{R}}{1-\mathbf{R}^{2} / L^{2}}$, where $k_{\mathrm{B}}$ is the Boltzmann constant, $T$ is temperature, and $\left\langle\mathbf{R}^{2}\right\rangle_{0} \simeq a_{s} L=a_{s}^{2} N$. The solution is characterized by the elastic modulus $G$ and the relaxation time $\tau$, and the viscosity is found from the scaling formula $\eta \simeq G \tau$. In the case of semidilute solutions without entanglements, $\tau \propto N^{2}$ (the Rouse time) and $G=n k_{\mathrm{B}} T$, where $n$ is the concentration of chains.

The rheological equation of the FENE-P model is formulated in terms of tensor $\mathbf{A}=\langle\mathbf{R} \mathbf{R}\rangle$, where the angle brackets denote averaging over the conformations of all chains. The stress tensor is determined by the formula

$$
\boldsymbol{\Sigma}=G \frac{\mathbf{A} / R_{0}^{2}-\mathbf{I}}{1-\operatorname{tr} \mathbf{A} / L^{2}},
$$

where the tensor of conformations $\mathbf{A}$ satisfies the equation

$$
\begin{gathered}
\tau\left[\frac{\partial \mathbf{A}}{\partial t}+(\mathbf{v} \cdot \boldsymbol{\nabla}) \mathbf{A}-(\nabla \mathbf{v})^{T} \cdot \mathbf{A}-\mathbf{A} \cdot \nabla \mathbf{v}\right] \\
+\frac{\mathbf{A}-R_{0}^{2} \mathbf{I}}{1-\operatorname{tr} \mathbf{A} / L^{2}}=0
\end{gathered}
$$

in which $R_{0}^{2}=\frac{1}{3}\left\langle\mathbf{R}^{2}\right\rangle_{0}$ and $\mathbf{v}$ is the velocity field. The velocity gradients are

$$
(\nabla \mathbf{v})_{i j}=\frac{\partial v_{j}}{\partial x_{i}}, \quad(\nabla \mathbf{v})_{i j}^{T}=\frac{\partial v_{i}}{\partial x_{j}}, \quad i, j=1,2,3 .
$$

Longitudinal electric field $E_{z}$ acting on the jet includes an external field $E_{0}$ and the field of charges of the cone-shaped meniscus $E_{\mathrm{c}}, E_{z}=E_{0}+E_{c}$. The contribution of the meniscus on scales larger than the transition region radius $b$ (usually $b<100 \mu \mathrm{m}$ ) can be represented as [19-21]

$$
E_{c}(z) \simeq \kappa \sqrt{\frac{\gamma}{\varepsilon_{0}(b+z)}} \equiv \frac{E^{*}}{\sqrt{1+z / b}} .
$$

Here the numerical coefficient $\kappa$ depends on the angle at the apex of the cone, and $z=0$ corresponds to the transition point between the cone and the jet $E_{c}(0)=E^{*}=\kappa \sqrt{\frac{\gamma}{\varepsilon_{0} b}}$. It is obvious that formula ceases to be valid on scales exceeding the size of the meniscus. In this region, the field is mainly defined by the electrodes. The formula for the total field $E_{z}$ gives the correct behavior near the transition region and at infinity. It will be used to estimate the field along the entire jet.

The electric current passing through the jet is described by the expression

$$
I \simeq I_{\mathrm{c}}+I_{\mathrm{b}} \simeq 2 \pi a \sigma_{\mathrm{f}} V_{z}+\pi a^{2} K E_{z},
$$

where $\sigma_{\mathrm{f}}$ is the surface charge density and currents $I_{\mathrm{b}}=I_{\mathrm{b}}(z)$ and $I_{\mathrm{c}}=I_{\mathrm{c}}(z)$ depend on $z$. At the point of transition of the cone to the jet, $I_{\mathrm{b}}(0)=I_{\mathrm{c}}(0)=I / 2$. The jet radius is calculated from the force balance equation [28]

$$
a^{2} \frac{d}{d z}\left(\gamma C-F_{n}\right)+\frac{d}{d z}\left[a^{2}\left(\rho v_{z}^{2}+\Sigma_{r r}-\Sigma_{z z}\right)\right]=2 a F_{z}
$$

$(C \simeq 1 / a$ is the general curvature of the surface $(a(0)=$ $b)$ ). As noted in the Introduction, the contribution of the normal component of the electric force $F_{\text {n }}$ to the overall balance of forces in the jet is relatively small; therefore, we will omit it below. The tangential component of the field acting per unit area of the jet surface is calculated in the standard way:

$$
F_{z}=\sigma_{f} E_{z} \text {. }
$$

We will assume that the value of current is known; therefore, the surface charge density $\sigma_{f}$ can be expressed in terms of current using formula (5). Eliminating stresses from Eq. (6) by formula (2) and taking into account that $\gamma \frac{d C}{d z} \simeq-\frac{\gamma}{a^{2}} \frac{d a}{d z}$, we have

$$
\begin{gathered}
\frac{d a}{d z}\left(\gamma+\frac{2 \rho Q^{2}}{\pi^{2} a^{3}}\right)+G \frac{d}{d z}\left[\frac{a^{2}\left(A_{z z}-A_{r r}\right) / R_{0}^{2}}{1-\left(A_{z z}+2 A_{r r}\right) / L^{2}}\right] \\
=-\frac{E_{z} a^{2}}{Q}\left(I-\pi a^{2} K E_{z}\right) .
\end{gathered}
$$

Here the components of the conformation tensor satisfy Eq. (3). Expressions (8) and (3) can be written in dimensionless form using the substitution

$$
a=b^{*} \bar{a}, \quad z=\ell \bar{z}, \quad \mathbf{A}=\overline{\mathbf{A}} R_{0}^{2},
$$


where $b^{*}=\gamma / G$ and $\ell=\frac{Q \tau}{\pi b^{*^{2}}}=\frac{Q \eta G}{\pi \gamma^{2}}$ are internal length scales. After some transformations, we get

$$
\begin{gathered}
\frac{d \bar{A}_{z z}}{d \bar{z}}+\frac{4 \bar{A}_{z z}}{\bar{a}} \frac{d \bar{a}}{d \bar{z}}+\frac{\bar{a}^{2}\left(\bar{A}_{z z}-1\right)}{1-\delta\left(\bar{A}_{z z}+2 \bar{A}_{r r}\right)}=0, \\
\frac{d \bar{A}_{r r}}{d \bar{z}}-\frac{2 \bar{A}_{r r}}{\bar{a}} \frac{d \bar{a}}{d \bar{z}}+\frac{\bar{a}^{2}\left(\bar{A}_{r r}-1\right)}{1-\delta\left(\bar{A}_{z z}+2 \bar{A}_{r r}\right)}=0, \\
\frac{d \bar{a}}{d \bar{z}}\left[1+\frac{\omega}{\bar{a}^{3}}-\frac{2 \bar{a}\left(\bar{A}_{z z}+2 \bar{A}_{r r}\right)}{1-\delta\left(\bar{A}_{z z}+2 \bar{A}_{r r}\right)}\right. \\
\left.-\frac{4 \delta \bar{a}\left(\bar{A}_{z z}-\bar{A}_{r r}\right)^{2}}{\left(1-\delta\left(\bar{A}_{z z}+2 \bar{A}_{r r}\right)\right)^{2}}\right]=\frac{\bar{a}^{4}\left(\bar{A}_{z z}-\bar{A}_{r r}\right)(1-3 \delta)}{\left(1-\delta\left(\bar{A}_{z z}+2 \bar{A}_{r r}\right)\right)^{3}} \\
-\chi \bar{a}^{2} \psi(\bar{z})\left(1-\frac{\bar{a}^{2} b^{* 2} \psi(\bar{z})}{2 b^{2} \psi(0)}\right) .
\end{gathered}
$$

In the above equations, the notation is as follows:

$$
\begin{gathered}
\delta=\frac{R_{0}^{2}}{L^{2}}=\frac{1}{3 N}, \quad \chi=\frac{\eta I E_{0}}{\pi \gamma^{2}}, \\
\omega=\frac{2 \rho Q^{2}}{\pi^{2} \gamma b^{*^{3}}}=\frac{2 \rho Q^{2} G^{3}}{\pi^{2} \gamma^{4}}, \\
\psi(\bar{z})=1+\frac{E^{*}}{E_{0}} \frac{1}{\sqrt{1+\ell \bar{z} / b}} .
\end{gathered}
$$

The numerical solution of the system of Eqs. (10)(12) will be carried out for chains with the number of segments $N=600$ and $N=2000$ and with ratio $E^{*} / E_{0}=2$. For chains with $N=600$ segments, we will use the following parameters: $\chi=2, \omega=0.0095$, $b / b^{*}=1 / \sqrt{20}$, and $\ell / b=0.2$. For chains with the number of segments $N=2000$, the parameters are as follows: $\chi=3, \omega=0.003, b / b^{*}=1 / \sqrt{40}$, and $\ell / b=0.1$. The indicated values are derived from the real values of the electrospinning parameters [1]. When choosing the values of the parameters, we also proceeded from the fact that the solutions of Eqs. (10)-(12) describe an infinitely long rectilinear jet.

Further, the external field strength increases by $v$ times, $E_{0} \rightarrow \vee E_{0}$. In the calculations, we assume that $v=2$. After increasing the field, the radius of the transition region $b$ and the field in the transition region $E^{*}$ change as follows: $b \rightarrow \beta b$ and $E^{*} \rightarrow E^{*} / \sqrt{\beta}$. Coefficient $\beta$ is found from the equality of the bulk and convective currents at the transition point $\pi \beta^{2} b^{2} K\left(v E_{0}+E^{*} / \sqrt{\beta}\right)=\frac{2 Q \sigma_{\mathrm{f}}^{*}}{\beta b}$, where $\sigma_{\mathrm{f}}^{*} \simeq \varepsilon_{0} E^{*} / \sqrt{\beta}$.

Using the latter equality for $v=1$ and arbitrary $v$, we can write the equation for $\beta$

$$
v \beta^{7 / 2}-1+\frac{E^{*}}{E_{0}}\left(\beta^{3}-1\right)=0 .
$$

Thus, the values of the parameters in formulas (10)(12), which depend on the external field, change as follows: $\chi \rightarrow \chi v / \beta^{3 / 2}, \quad E^{*} / E_{0} \rightarrow\left(E^{*} / E_{0}\right) /\left(v \beta^{1 / 2}\right)$, $\bar{a}(0) \rightarrow \bar{a}(0) \beta$, and $\ell / b \rightarrow(\ell / b) / \beta$. At the point $z=0$, the conformation of polymer chains is assumed to be Gaussian, $\bar{A}_{z z}(0)=\bar{A}_{r r}(0)=1$, and the radius of the jet is $\bar{a}(0)=b / b^{*}$. For the given parameters, the inertial force and the viscoelastic force in the transition region are comparable with the capillary force. Differential equations (10)-(12) with initial conditions were solved using the Runge-Kutta method.

\section{RESULTS AND DISCUSSION}

Relations (10)-(12) also make it possible to assess the orientation of the chains depending on the coordinate $\bar{z}$ characterized by the order parameter $s=\sqrt{\left(A_{z z}-R_{0}^{2}\right)} / L=\sqrt{\delta\left(\bar{A}_{z z}-1\right)}$, which varies within $0 \leq s \leq 1$. For highly stretched chains, $s \simeq R_{z} / L$, where $R_{z}$ is the size of the chain along the jet axis [1618].

Calculations show that two modes of jet behavior are possible, the transition between which occurs when the value of the external electric field changes by $v$ times $(v=2)$. In weak fields, the jet remains rectilinear and infinitely long (mode I). Plots of functions $\bar{a}(\bar{z}), s(\bar{z})$, and $\bar{A}_{r r}(\bar{z})$ for $N=600$ and $N=2000$ are shown in Figs. 2a and Fig. 3a, respectively. The radius of the jet decreases monotonically, and the order parameter $s(\bar{z})$ first increases along the jet axis and after reaching the maximum value begins to decrease. Component $\bar{A}_{r r}(\bar{z})$ of the conformation tensor also behaves nonmonotonically: initially it decreases and then increases to the equilibrium value corresponding to the state of the coil. This behavior indicates the occurrence of the processes of relaxation of chains after their stretching and longitudinal compression. It is interesting to note that the relaxation of the transverse size of macromolecules begins earlier than that of the longitudinal one.

After a twofold increase in the field strength, the behavior of the jet changes qualitatively: the jet remains rectilinear only in the end segment (regime II). At the end of the segment, its behavior becomes singular $\frac{d \bar{a}}{d \bar{z}} \rightarrow-\infty$. The behavior of functions $\bar{a}(\bar{z})$, $s(\bar{z})$, and $\bar{A}_{r r}(\bar{z})$ in this case is illustrated by Fig. $2 \mathrm{~b}$ and Fig. 3b. The order parameter monotonically increases along the jet axis and reaches its maximum value at the end of the straight section. The jet radius is reduced. Function $\bar{A}_{r r}(\bar{z})$ first decreases and then increases. The appearance of the singularity is associated with 

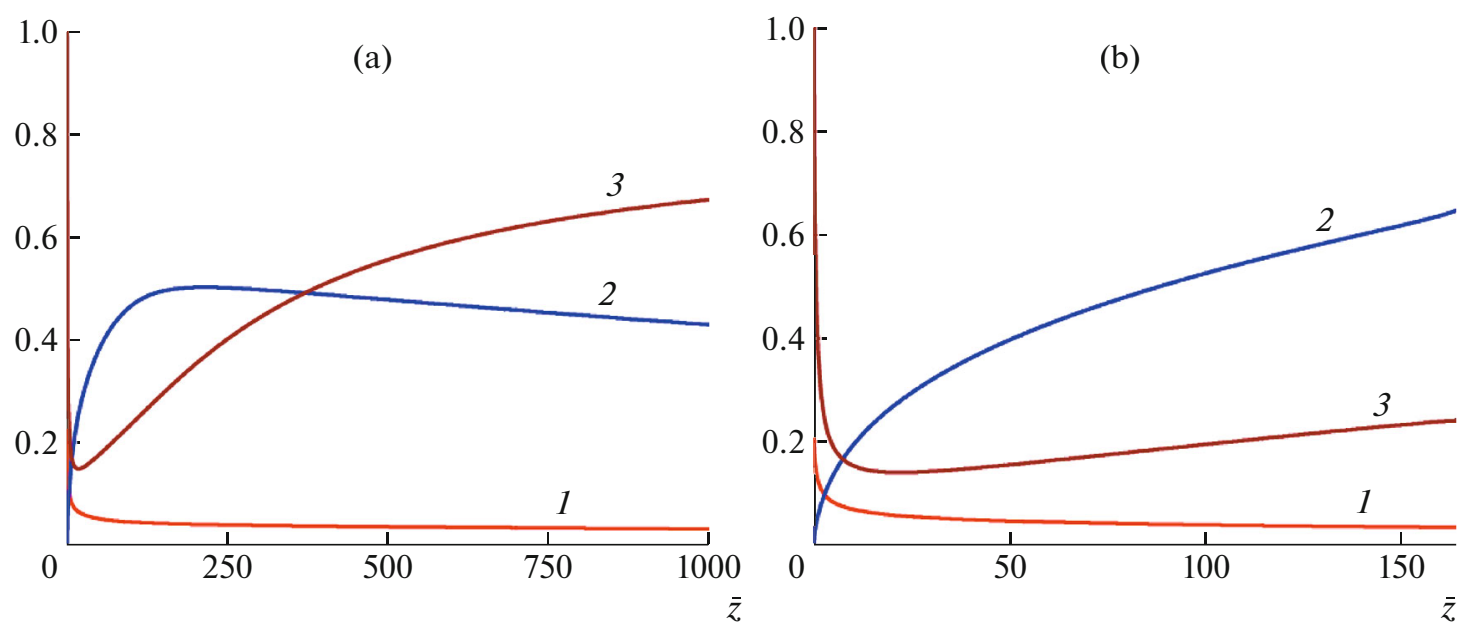

Fig. 2. Dependence of (1) jet radius $\bar{a}$, (2) order parameter $s$, and (3) component $\bar{A}_{r r}$ of the conformation tensor on the coordinate $\bar{z}$ along the jet axis for $N=600$ and $\omega=0.0095$. (a) Mode I and (b) mode II.
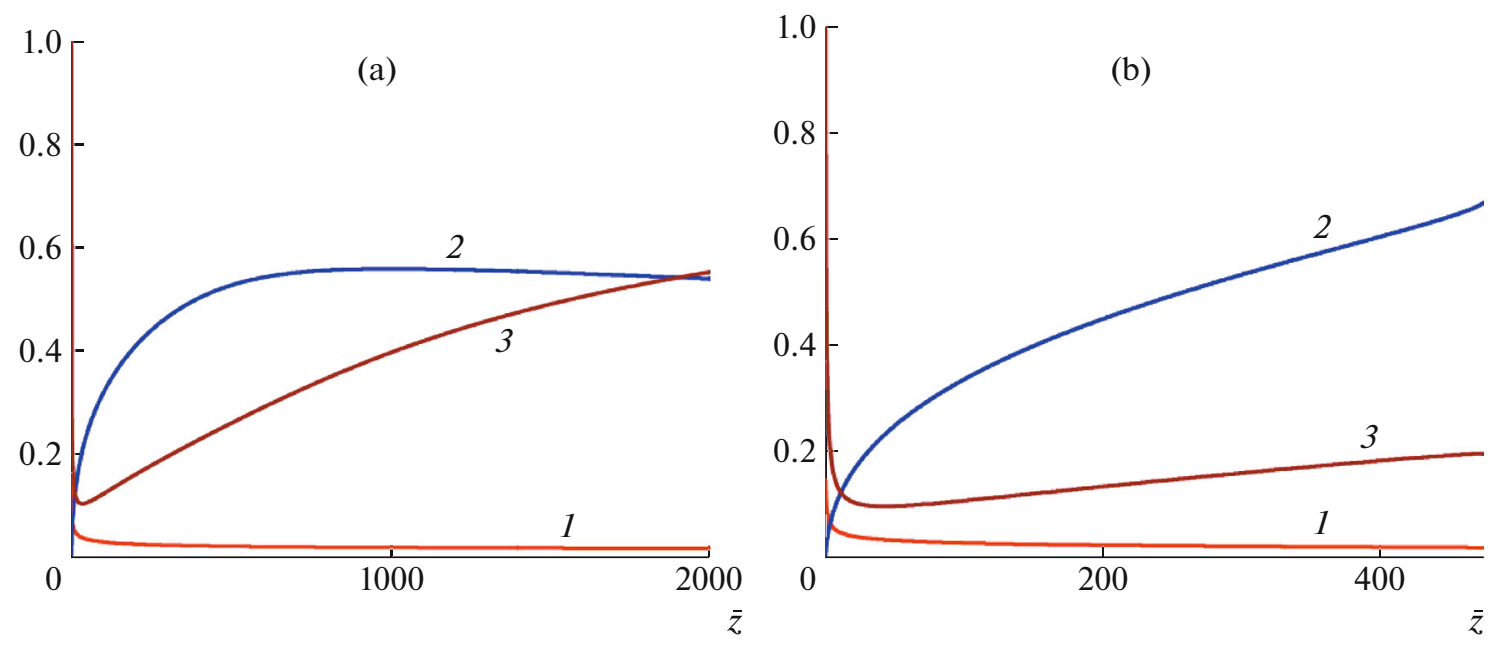

Fig. 3. Dependence of (1) jet radius $\bar{a}$, (2) order parameter $s$, and (3) component $\bar{A}_{r r}$ of the conformation tensor on the coordinate $\bar{z}$ along the jet axis for $N=2000$ and $\omega=0.003$. (a) Mode I and (b) mode II.

the finite extensibility of the chains, and mathematically, this occurs due to the vanishing of the sum in brackets on the left-hand side of Eq. (12). After passing through the singularity point, the motion of the jet should be unstable: the most probable scenario is bending of the jet. Obviously, the thin body approximation ceases to work here.

For a more detailed analysis of the jet dynamics, the dependences of the Weissenberg number $\mathrm{Wi}=$ $\dot{\varepsilon} \tau=\frac{2}{\bar{a}^{3}}\left|\frac{d \bar{a}}{d \bar{z}}\right|$ on coordinate $\bar{z}$ (Fig. 4), the value of $\bar{A}_{z z}^{1 / 4}$ on $1 / \bar{a}$ (Fig. 5), and the flow rate $\bar{V}_{z}(\bar{z})=\frac{V_{z} \tau}{\ell}=\frac{1}{\bar{a}^{2}}$ on $\bar{z}^{1 / 2}$ were considered (Fig. 6). Calculations indicate that the Weissenberg number in the transition region is large, Wi $\gg 1$, which is in agreement with experiment [3-5]. Then it begins to decrease with distance from the transition region (Figs. 4a, 4b). A drop in the values of Wi always occurs in mode I, where the electric field is weak (Fig. 4, curves 1). However, after a twofold increase in the external field strength (mode II), the picture is qualitatively different and a decrease in the Weissenberg number gives way to its rapid growth (Fig. 4, curves 2). This is due to the approach to the singularity point. In this case, the Weissenberg number is always greater than one, $\mathrm{Wi}>1$.

At high Weissenberg numbers $(\mathrm{Wi}>1)$, the solution should be deformed elastically. To verify this fact, we considered the dependence of the value of $\bar{A}_{z z}^{1 / 4}$ on $1 / \bar{a}$, which under elastic deformation (high elasticity 

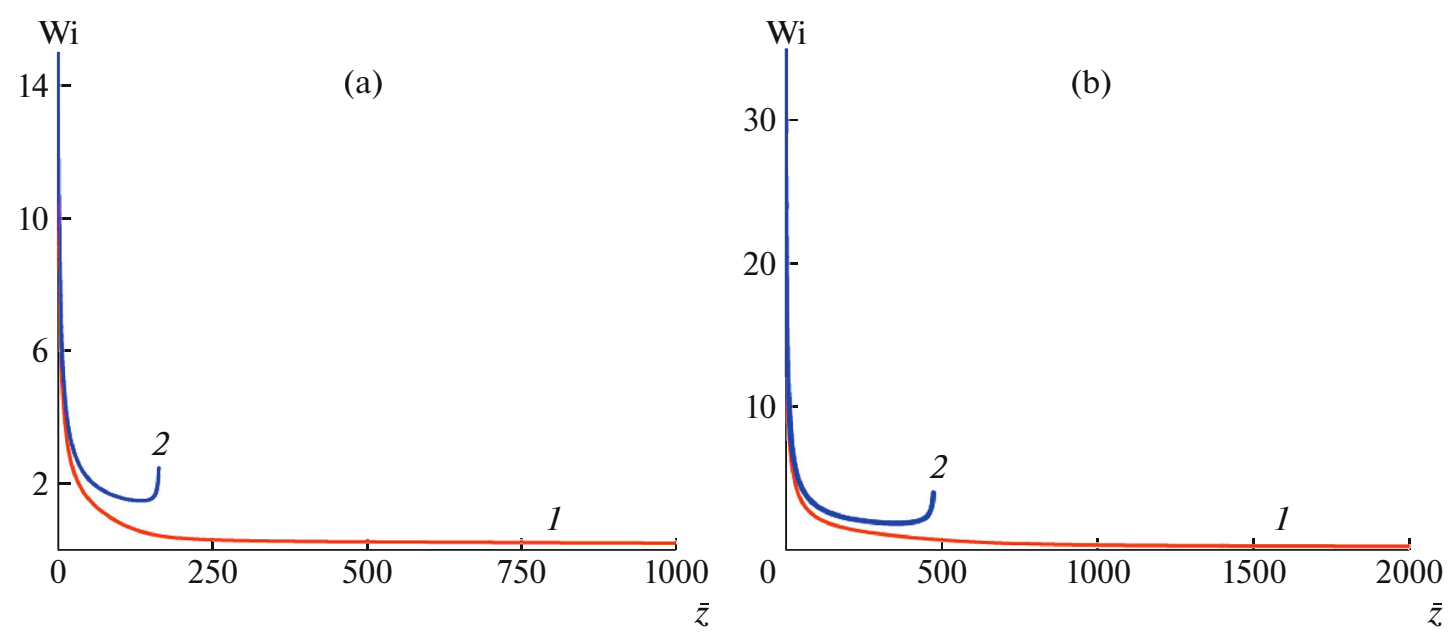

Fig. 4. Dependence of the Weissenberg number Wi on the coordinate $\bar{z}$. (a) $N=600$ and $\omega=0.0095$, and (b) $N=2000$ and $\omega=0.003$. Curve 1 corresponds to the infinitely long rectilinear jet, and curve 2 corresponds to the jet with the limited straight segment.
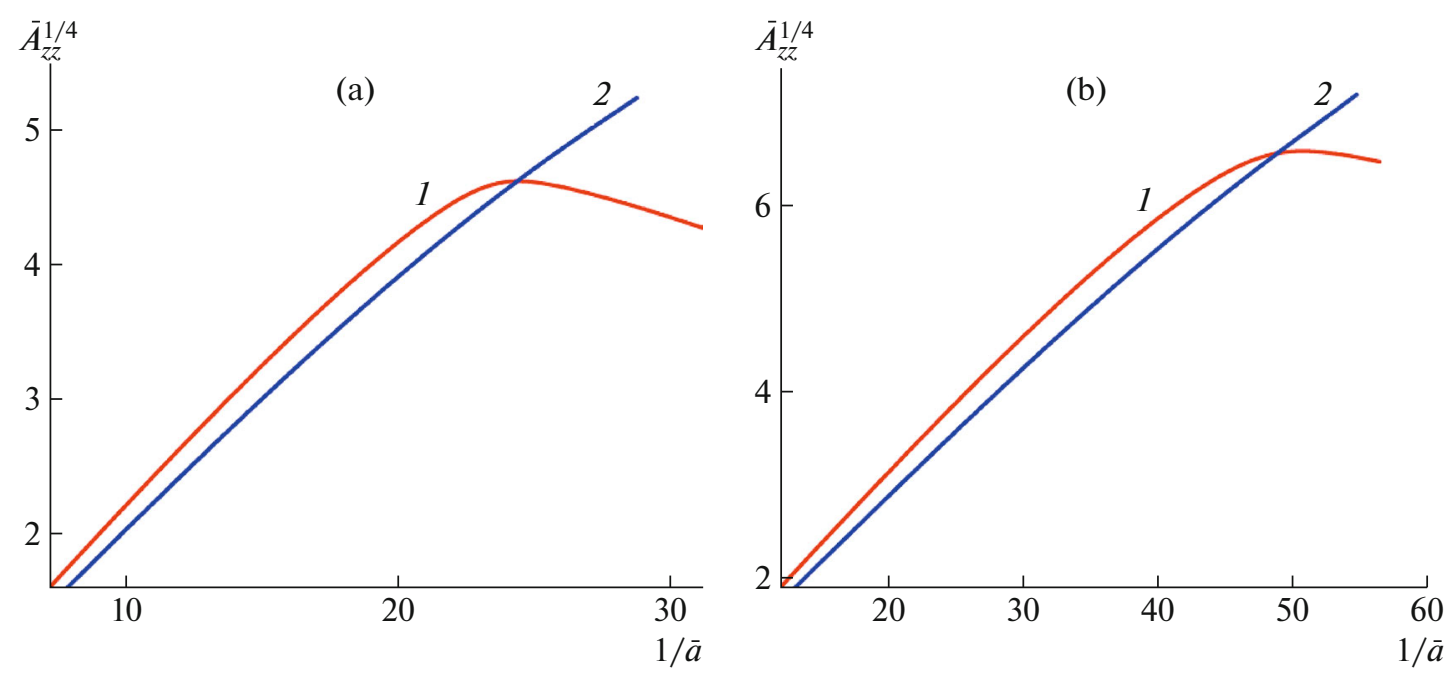

Fig. 5. Dependence of the value of $\bar{A}_{z z}^{1 / 4}$ on $1 / \bar{a}$. (a) $N=600$ and $\omega=0.0095$, and (b) $N=2000$ and $\omega=0.003$. Curve 1 corresponds to the infinitely long rectilinear jet, and curve 2 corresponds to the jet with the limited straight segment.

region) should be linear. Indeed, if we take a small section of the jet of length $\Delta \bar{z}_{0}$ and volume $\pi \bar{a}_{0}^{2} \Delta \bar{z}_{0}$ inside the transition region, then owing to the conservation of volume its length after moving at a certain distance will be $\Delta \bar{z}=\frac{\bar{a}_{0}^{2}}{\bar{a}^{2}} \Delta \bar{z}_{0}$, where $\bar{a}$ is the current radius. Thus, the longitudinal size of the polymer chains increases by $\bar{a}_{0}^{2} / \bar{a}^{2}$ times [24]. Since $\bar{A}_{z z}$ is proportional to the square of the longitudinal dimension of the chain, the estimate $\bar{A}_{z z} \simeq \bar{a}_{0}^{4} / \bar{a}^{4}$ must be valid. Figure 5 shows that the function $\bar{A}_{z z}^{1 / 4}$ depends on $1 / \bar{a}$ almost linearly, as in mode I until the chains reach their maximum length (curves 1) and in mode II (curves 2). Thus, the order parameter changes according to the law $s \simeq \bar{a}_{0}^{2} \sqrt{\delta} / \bar{a}^{2}$.
During elastic deformation of the solution when the chains are not yet too strongly stretched, the viscoelastic forces change in almost the same way as the inertial forces but with the opposite sign. This follows from the left-hand side of Eq. (12), where the second and third terms in parentheses show the same dependence on the jet radius $\sim 1 / \bar{a}^{3}$. It is obvious that the fourth term, which is also related to viscoelasticity, should be fairly small. This behavior also affects the flow rate in the jet. The dependence of the dimensionless velocity on the distance is shown in Fig. 6. It can be seen that, in mode II (curves 2), the velocity is almost linearly dependent on the coordinate and, in mode I (curves 1), two regions can be distinguished in which the velocity varies almost linearly with the coordinate, but the slope of the lines is different. This is due to the fact that, in the region close to the transition 

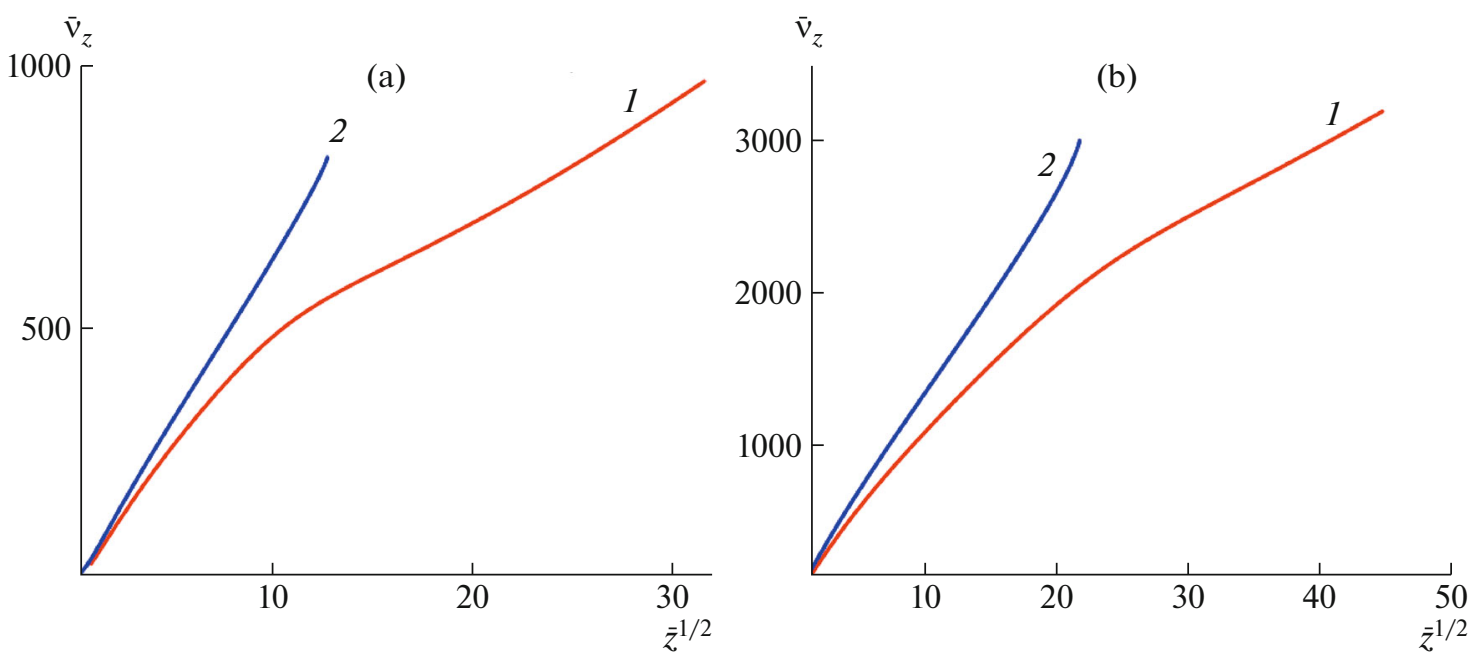

Fig. 6. Jet velocity as a function of $\sqrt{\bar{z}}$. (a) $N=600$ and $\omega=0.0095$, and (b) $N=2000$ and $\omega=0.003$. Curve 1 corresponds to the infinitely long rectilinear jet, and curve 2 corresponds to the jet with the limited straight segment.

zone, the inertia is partially compensated by the viscoelasticity, while in the far zone the contribution of viscoelastic forces is fairly small. Thus, the velocity and radius of the jet change like this: $\bar{v}_{z}(\bar{z}) \propto \bar{z}^{1 / 2}$ and $\bar{a}(\bar{z}) \propto \bar{z}^{-1 / 4}$, which is consistent with the dependences of the velocity on the axial coordinate obtained experimentally [35].

\section{CONCLUSIONS}

The study of changes in the conformations of polymer chains in the jet by the numerical analysis of the force balance equation and using the rheological equation of the model of finitely extensible chains (FENE-P) makes it possible to assess the relationship between the orientation of the chains and the dynamics of the jet and to analyze the role of nonlinear viscoelasticity associated with the finite length and its correlation with capillary and inertial forces. It is shown that polymer chains in the jet can stretch strongly along the stretching axis. A new type of jet instability is discovered, which differs from the Rayleigh instability, as well as from the axisymmetric and nonaxisymmetric instabilities arising owing to repulsion between charges on the jet surface [1, 2, 41-44]. At a fixed volume flow rate of the liquid, this instability appears with an increase in the external field strength, when the Weissenberg number $\mathrm{Wi}>1$ and the solution is in the highly elastic region. In this mode, the polymer chains are monotonically stretched to a certain maximum value, after which their further stretching becomes impossible and the jet can no longer remain rectilinear. In this case, the Weissenberg number changes nonmonotonically: first it decreases and then begins to grow. This behavior is consistent with the dependence of the stretching rate on the axial coordinate experimentally reported in [36]. The orienta- tional order parameter defined as the ratio of the longitudinal size of the chain to its contour length monotonically increases along the jet axis as $s \simeq \bar{a}_{0}^{2} \sqrt{\delta} / \bar{a}^{2} \propto(z / N)^{1 / 2}$ and reaches its maximum value at the end of the straight section of the jet. Further motion of the jet becomes unstable, and it begins to bend: in this case, the relaxation of polymer chains occurs.

In fairly weak electric fields, the role of inertia is decisive and the jet always remains rectilinear. In this mode, the orientational order parameter changes along the jet nonmonotonically: first it increases to a certain maximum value (the Weissenberg number decreases to $\mathrm{Wi} \sim 1$ ) and then decreases. The decrease in the order parameter is due to the relaxation of polymer chains and is characterized by the Rouse time. Thus, the dynamics of growth and relaxation of the order parameter slows down with increasing molecular weight of the polymer.

The transition from the rectilinear jet to the jet in which whipping instability arises with an increase in the external electric field strength was experimentally investigated, for example, in the electrospinning of $2 \%$ PEO aqueous solutions [48, 49] and concentrated solutions of polystyrene in dimethylformamide [50]. On the basis of the results obtained, it can be assumed that the cause of the bending instability of the rectilinear jet in strong fields can be associated not only with the repulsion between charges on its surface $[1,43]$ but also with its high elasticity and orientation of macromolecules along the jet axis.

\section{FUNDING}

This study was supported by the Russian Science Foundation (project no. 20-19-00194). 


\section{OPEN ACCESS}

This article is distributed under the terms of the Creative Commons Attribution 4.0 International license (http://creativecommons.org/licenses/by/4.0/), which permits unrestricted use, distribution, and reproduction in any medium, provided you give appropriate credit to the original author(s) and the source, provide a link to the Creative Commons license, and indicate if changes were made.

\section{REFERENCES}

1. D. H. Reneker, A. L. Yarin, E. Zussman, and H. Xu, Adv. Appl. Mech. 41, 43 (2007).

2. M. Lauricella, S. Succi, E. Zussman, D. Pisignano, and A. L. Yarin, Rev. Mod. Phys. 92, 035004 (2020).

3. J. Xue, T. Wu, Y. Dai, and Y. Xia, Chem. Rev. 119, 5298 (2019).

4. Energy Harvesting Properties of Electrospun Nanofibers, Ed. by J. Feng and T. Lin (IOP Publ., Bristol, 2020).

5. C. Wang and T. Hashimoto, Macromolecules 51, 4502 (2018).

6. Y. Wang and C. Wang, Macromol. Mater. Eng. 304, 1900281 (2019).

7. T. Hashimoto, Y. Wang, H-Y. Lai, and C.-H. Kuo, Macromolecules 53, 7876 (2020).

8. M. V. Kakade, S. Givens, K. Gardner, K. H. Lee, D. B. Chase, and J. F. Rabolt, J. Am. Chem. Soc. 129, 2777 (2007).

9. M. Richard-Lacroix and C. Pellerin, Macromolecules 48, 4511 (2015).

10. Z. Wang, B. Sun, X. Lu, C. Wang, and Z. Su, Macromolecules 52, 9639 (2019).

11. A. V. Bazilevskii, S. I. Voronkov, V. M. Entov, and A. N. Rozhkov, Sov. Phys. Dokl. 26, 333 (1981).

12. R. Sattler, S. Gier, J. Eggers, and C. Wagner, Phys. Fluids 24, 023101 (2012).

13. A. V. Semakov, V. G. Kulichikhin, A. K. Tereshin, S. V. Antonov, and A. Ya. Malkin, J. Polym. Sci., Part B: Polym. Phys. 53, 559 (2015).

14. A. Ya. Malkin, A. V. Semakov, I. Yu. Skvortsov, P. Zatonskikh, V. G. Kulichikhin, A. V. Subbotin, and A. N. Semenov, Macromolecules 50, 8231 (2017).

15. V. G. Kulichikhin, I. Yu. Skvortsov, A. V. Subbotin, S. V. Kotomin, and A. Ya. Malkin, Polymers 10, 856 (2018).

16. A. V. Subbotin and A. N. Semenov, J. Polym. Sci., Part B: Polym. Phys. 54, 1066 (2016).

17. A. N. Semenov and A. V. Subbotin, J. Polym. Sci., Part B: Polym. Phys. 55, 623 (2017).

18. A. V. Subbotin and A. N. Semenov, Polym. Sci., Ser. C 60, 106 (2018).

19. J. Fernández de la Mora, Annu. Rev. Fluid Mech. 39, 217 (2007).

20. J. Fernández de la Mora, J. Fluid Mech. 243, 561 (1992).

21. J. Fernández de la Mora and I. G. Loscertales, J. Fluid Mech. 260, 155 (1994).
22. F. J. Higuera, J. Fluid Mech. 484, 303 (2003).

23. A. M. Gañán-Calvo, J. Fluid Mech. 507, 203 (2004).

24. T. Han, A. L. Yarin, and D. H. Reneker, Polymer 49, 1651 (2008).

25. J. H. Yu, S. V. Fridrikh, and G. C. Rutledge, Polymer 47, 4789 (2006).

26. Y. Wang, T. Hashimoto, C.-C. Li, Y.-C. Li, and C. Wang, J. Polym. Sci., Part B: Polym. Phys. 56, 319 (2018).

27. S. N. Reznik and E. Zussman, Phys. Rev. E: Stat., Nonlinear, Soft Matter 81, 026313 (2010).

28. A. V. Subbotin and A. N. Semenov, Proc. R. Soc. London, Ser. A 471, 20150290 (2015).

29. A. V. Subbotin and A. N. Semenov, JETP Lett. 102, 815 (2015).

30. N. Kirichenko, I. V. Petryanov-Sokolov, N. N. Suprun, and A. A. Shutov, Sov. Phys. Dokl. 31, 611 (1986).

31. M. M. Hohman, M. Shin, G. Rutledge, and M. P. Brenner, Phys. Fluids 13, 2221 (2001).

32. J. J. Feng, Phys. Fluids 14, 3912 (2002).

33. C. P. Carroll and Y. L. Joo, Phys. Fluids 18, 053102 (2006).

34. A. Subbotin and V. G. Kulichikhin, Polym. Sci., Ser. A 56, 211 (2014).

35. M. E. Helgeson, K. N. Grammatikos, J. M. Deitzel, and N. J. Wagner, Polymer 49, 2924 (2008).

36. I. Greenfeld, K. Fezzaa, M. H. Rafailovich, and E. Zussman, Macromolecules 45, 3616 (2012).

37. D. H. Reneker and A. L. Yarin, Polymer 49, 2387 (2008).

38. A. I. Zhakin, Phys.-Usp. 56, 141 (2013).

39. C. Wang, Y.-W. Cheng, C.-H. Hsu, H.-C. Chien, and S.-Y. J. Tsou, Polym. Res. 18, 111 (2011).

40. S. L. Shenoy, W. D. Bates, H. L. Frisch, and G. E. Wnek, Polymer 46, 3372 (2005).

41. C. P. Carroll and Y. L. Joo, J. Non-Newtonian Fluid Mech. 153, 130 (2008).

42. D. Deshawar and P. Chokshi, Polymer 131, 34 (2017).

43. D. H. Reneker, A. L. Yarin, H. Fong, and S. Koombhongse, J. Appl. Phys. 87, 4531 (2000).

44. S. P. Shariatpanahi, D. Bonn, M. R. Ejtehadi, and A. I. Zad, J. Polym. Sci., Part B: Polym. Phys. 54, 1036 (2016).

45. R. G. Larson and P. S. Desai, Annu. Rev. Fluid Mech. 47, 47 (2015).

46. A. V. Bazilevskii and A. N. Rozhkov, Fluid Dyn. 49, 827 (2014).

47. P. Szabo, G. H. McKinley, and C. Clasen, J. NonNewtonian Fluid Mech. 169-170 (2), 26 (2012).

48. Y. M. Shin, M. M. Hohman, M. P. Brenner, and G. C. Rutledge, Appl. Phys. Lett. 78, 1149 (2001).

49. Y. M. Shin, M. M. Hohman, M. P. Brenner, and G. C. Rutledge, Polymer 42, 9955 (2001).

50. Y. Xin and D. H. Reneker, Polymer 53, 4254 (2012). 